\title{
Las nuevas reformas educativas en el Ecuador y su aplicación en educación especial
}

\section{The new reform educational in the Ecuador and its application in education special}

\author{
Rocío Ernestina García Liscano*, Verónica Alexandra García Liscano ${ }^{\dagger}$ \\ Universidad Técnica de Babahoyo - Extensión Quevedo, Ecuador. \\ \{lexislex_rocio;garlis5vero\}@ $@$ hotmail.com
}

Fecha de recepción: 28 de noviembre de 2016 - Fecha de aceptación: 3 de marzo de 2017

\begin{abstract}
Resumen-El Ecuador con el paso de los años, el gobierno de la revolución ciudadana ha realizado cambios drásticos dentro del sistema de educación, con las finalidad de mejorar la calidad de aprendizaje de los ecuatorianos. El presente artículo está orientado a analizar la reforma educativa en el Ecuador a partir de la nueva institucionalidad del país, la que se constituye en el gobierno del Econ. Rafael Correa Delgado con el nuevo modelo Educativo que se inició en enero de 2010, a partir de esta restructuración realizamos un análisis de la educación especial en nuestro país, si se ha considerado dentro de las reformas realizadas y si se toma la debida importancia referente a la preparación de personas discapacitadas. Este análisis contribuye también a demostrar cuales son los beneficios que ha generado la reforma educativa dentro de las unidades educativas especializadas, tomando como base la perspectiva del antes y después, y como mediante, su aplicación se ha logrado fortalecer los procesos de mejoramiento de la calidad, relevancia, equidad y eficiencia de la educación especial en el Ecuador.
\end{abstract}

Palabras Clave-Reformas Educativas, Educación Especial, Inclusión Educativa.

Abstract-The Ecuador over the years, the government of the citizen revolution has made drastic changes in the education system, with the aim of improving the quality of learning of Ecuadorians. This article is aimed at analyzing the educational reform in Ecuador from the new institutions of the country, which constitutes the government of Econ. Rafael Correa Delgado with the new educational model that began in January 2010, from this restructuring conducted an analysis of special education in our country, if considered in the reforms and if taken due importance concerning the preparation of disabled people. This analysis also helps to demonstrate what are the benefits generated by the educational reform within specialized educational units, based on the perspective of before and after, and as by their application has been made to strengthen the processes of quality improvement, relevance, equity and efficiency of special education in Ecuador.

Keywords-Educational reforms, Ecuadorian Education, Educational inclusion.

\section{INTRODUCCIÓN}

$\mathbf{L}$ os centros escolares constituyen, en nuestros días, contextos caracterizados por la diversidad de su alumnado, reflejo a su vez, de sociedades cada vez más diversas. El cuerpo de estudiantes es hoy más heterogéneo que nunca en su composición cultural, étnica, lingüística, de clase, de género, de capacidad.

Cuando se habla de reforma educativa se alude a las decisiones que toma un Estado con el fin de orientar políticas educativas para superar las deficiencias detectadas en un sistema con resultados insatisfactorios en términos de calidad y eficiencia. Toda reforma educativa es producto de una gestión que evalúa cómo el servicio educativo que brinda a sus ciudadanos influye positiva o negativamente en la estructura social y educativa de un país determinado (Pombo et al., 2015).

La Educación Especial es un servicio diferencial en el contexto de la Educación Regular. Atiende a niños, adolescentes, jóvenes y adultos con características biológicas, psíquicas y socio-culturales diferentes, como consecuencia de antecedentes patológicos o clínicos, o de privaciones socioeconómicas y

\footnotetext{
*Ingeniera en Gestión Empresarial, Magister en Docencia y Currículo.

${ }^{\dagger}$ Licenciada en Ciencias de la Educación mención Idiomas, Magister en Docencia y Currículo.
}

culturales. No es una instancia marginal, en virtud de que su función es incorporar al alumno, en el menor tiempo posible, a la educación común (Almeida, 2012).

El derecho a la educación que asiste a todos por igual, sin discriminaciones, es el principio rector de la política educacional, que se pretende sea una realidad con la participación consciente y dinámica de la familia, la sociedad civil en su conjunto y las organizaciones gubernamentales creadas para el efecto.

La Educación Especial es una modalidad de atención del sistema educativo de tipo transversal e interdisciplinario dirigida a estudiantes con necesidades educativas especiales asociadas la discapacidad no susceptible de inclusión. Plantea el reconocimiento de las diferencias y el respeto de la diversidad, asegura el cumplimiento del principio de equiparación de oportunidades, como un aspecto de enriquecimiento. Se caracteriza por proveer un conjunto de servicios, recursos humanos y técnicos, conocimientos especializados y ayudas, con el propósito de asegurar aprendizajes de calidad a los estudiantes (Ministerio de Educación, 2007).

\section{La Inclusión Educativa}

El interés por el fenómeno de la diversidad y sus implicaciones, tanto en el plano social, político y económico como, más 
específicamente, en el educativo y escolar en lo que respecta al currículo y enseñanza a desarrollar en las escuelas, y en lo que se refiere a los aspectos y dinámicas organizativas es evidente en los últimos años. Múltiples experiencias, reflexiones e investigaciones han ido abordando en el tema desde óptica de la exclusión educativa que amenaza a un buen número de alumnos en las instituciones educativas de nuestro país, desde la perspectiva de la inclusión educativa, así como desde los planeamientos de la justicia social, particularmente en sus versiones más críticas (González, 2008).

En la actualidad, nuestro país se enfrenta al reto de garantizar una educación de calidad con un enfoque inclusivo que se fundamenta en el principio de igualdad de oportunidades para todos, buscando superar la discriminación y favoreciendo a la diversidad. Por lo que consideramos que la educación con fundamento humanístico y las normas legales son el medio más eficaz para lograr un cambio y promover una sociedad justa y equitativa en la que se garantice los derechos humanos de todos y todas sin ningún tipo de discriminación (Montenegro and Tigre, 2013).

\section{Constitución de la República del Ecuador:}

Según la Constitución de la República del Ecuador en el Art. 3 numeral " 1 " señala que es deber del Estado "Garantizar sin discriminación alguna el efectivo goce de los derechos establecidos en la Constitución y en los instrumentos internacionales, en particular la educación, la salud, la alimentación, la seguridad social y el agua para sus habitantes.", y en el Art. 26, determina qué; "La educación es un derecho de las personas a lo largo de su vida y un deber ineludible e inexcusable del Estado. Constituye un área prioritaria de la Política Pública y la inversión estatal, garantía de la igualdad e inclusión social y condición indispensable para el buen vivir" (Constitución del Ecuador, 2012).

Por otro lado en el Art. 27, establece que; "La educación se centrará en el ser humano y garantizará su desarrollo holístico, en el marco del respeto a los derechos humanos, al medio ambiente sustentable y a la democracia; será participativa, obligatoria, intercultural, democrática, incluyente y diversa, de calidad y calidez; impulsará la equidad de género, la justicia, la solidaridad y la paz; estimulará el sentido crítico, el arte y la cultura física, la iniciativa individual y comunitaria, y el desarrollo de competencias y capacidades para crear y trabajar" (Constitución del Ecuador, 2012).

Según como se cita en la constitución del ecuador, el Estado tiene la obligación de proveer a todas las personas de manera ineludible y sin evasiva alguna y como una garantía del buen vivir el libre derecho a la educación, con igualdad de condiciones para todos sus habitantes del territorio ecuatoriano (Figura 1), facilitándoles a los mismos conocer sus derechos fundamentales, que permitan el desarrollo integral, claro está dentro de un marco democrático, entendiéndose a este como la posibilidad de participación sin distinciones sociales ni de condición alguna, sean estas de raza, cultura, económica, religiosa, política o de capacidad (María et al., 2015).

Vemos entonces que nuestro gobierno coloca el derecho a la educación como un derecho fundamental a todo ser humano y

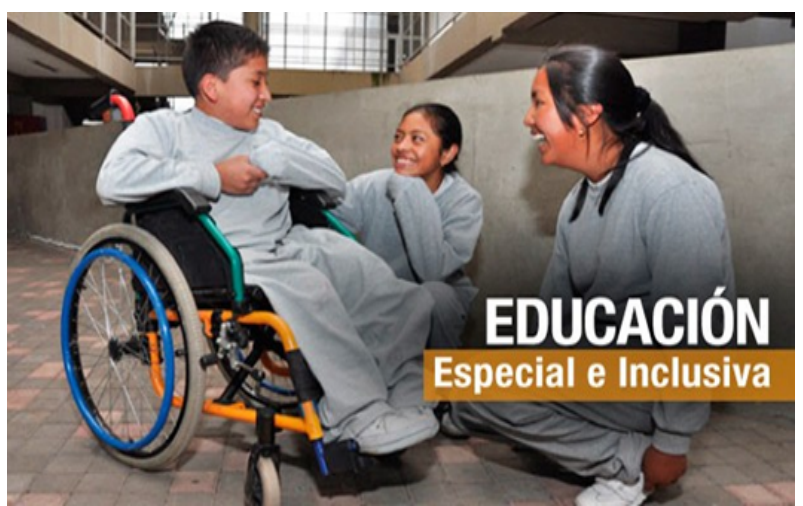

Figura 1. Educación Especial e Inclusiva

Fuente: (Educación Especial e Inclusiva - Ministerio de Educación, 2015)

en particular a todos quienes formamos parte de este país, vale la pena recalcar que respecto a esto se están dando los primeros pasos con un verdadero compromiso hacia un efectivo cambio de la forma de concebir a la educación no como un derecho de unos pocos, sino de toda la población en su conjunto, sin diferencias de ninguna clase. Se regula el acceso a la educación de todas las personas sin discriminación alguna, puesto que la educación debe responder al interés general de la población; facilitando que las diversas culturas existentes en el país interactúen, posibilitando el intercambio de conocimientos, lo que enriquece a la sociedad en su conjunto y garantiza que ninguno de sus miembros se sientan excluidos debido a la cultura en la que se han desarrollado (Montenegro and Tigre, 2013). En el Art.46, numeral "3" del Capítulo III sobre los Derechos de las Personas y Grupos de Atención Prioritaria, se manifiesta que se dará "atención preferente para la plena integración social de quienes tengan discapacidad (Constitución del Ecuador, 2012).

Como se citó anteriormente cada uno de los seres humanos gozamos de los mismos derechos con la diferencia de aquellos que tienen capacidades diferentes, ya que son ellos quienes tienen preferencia y gozan de garantías para su incorporación de manera total dentro del sistema de educación con la que cuenta nuestro país, recibiendo protección en todo aspecto y momento.

\section{Informe de unidades educativas especializadas en Ecuador}

En el Ecuador existen 100 unidades de educación especial. A continuación mostramos el número de unidades educativas especiales que existen por zona.

Como podemos ver en la figura 2 la en la zona 5 compuesta por las provincias de Los Ríos, Bolívar, Galápagos y El Guayas (El Triunfo) cuentan con 14 unidades educativas especializadas. Y en la tabla 1 se muestra los datos de las 9 unidades educativas que pertenecen a la provincia de Los Ríos. 


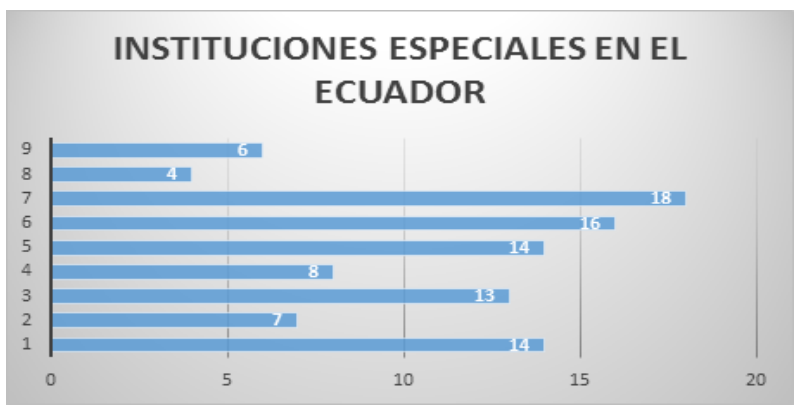

Figura 2. Unidades educativas de educación especial por zonificación Fuente: (Dirección Nacional de Educación Especializada e Inclusiva, 2014).

Tabla 1. Unidades Educativas de la provincia de los Ríos

\begin{tabular}{|l|l|l|}
\hline \multicolumn{1}{|c|}{ CANTON } & \multicolumn{1}{|c|}{ PARROQUIA } & NOMBRE DE IE \\
\hline MONTALVO & MONTALVO & $\begin{array}{l}\text { LEOPOLDO ZUMBA } \\
\text { ALTAMIRANO }\end{array}$ \\
\hline VINCES & VINCES & $\begin{array}{l}\text { ESCUELA DE EDUCACIÓN } \\
\text { ESPECIAL 3 DE DICIEMBRE }\end{array}$ \\
\hline VENTANAS & VENTANAS & VENTANAS \\
\hline QUEVEDO & QUEVEDO & PAMUNIQ \\
\hline PALENQUE & PALENQUE & $\begin{array}{l}\text { ESCUELA DE EDUCACIÓN } \\
\text { ESPECIAL CIUDAD DE } \\
\text { PALENQUE }\end{array}$ \\
\hline VALENCIA & VALENCIA & $\begin{array}{l}\text { ESCUELA ESPECIAL } \\
\text { FANADV }\end{array}$ \\
\hline PUEBLO VIEJO & PUEBLO VIEJO & PUEBLO VIEJO \\
\hline PUEBLO VIEJO & SAN JUAN & $\begin{array}{l}\text { ESCUELA DE EDUCACIÓN } \\
\text { ESPECIAL CARLOS ORTEGA } \\
\text { GONZÁLES }\end{array}$ \\
\hline BABAHOYO & $\begin{array}{l}\text { DR. CAMILO } \\
\text { PONCE }\end{array}$ & AYÚDANOS A EMPEZAR \\
\hline
\end{tabular}

Fuente: Dirección Nacional de Educación Especializada en Inclusiva, 2014.

\section{Metodología}

En el campo de la investigación existen muchos métodos que pueden aplicarse, sin embargo para el desarrollo de este trabajo utilizaremos el método inductivo intenta ordenar la observación tratando de extraer conclusiones de carácter universal desde la acumulación de datos particulares. La técnica que se utiliza para el desarrollo de esta investigación es la entrevista ya que esta permitirá indagar sobre la problemática más a fondo y directamente conocer los hechos desde los actores principales en el tema de investigación.

\section{La entrevista}

En la ciudad de Babahoyo en las calles Vargas Machuca y la $\mathrm{K}$ en la ciudadela Barrio lindo se encuentra ubicada la Escuela de Educación Especial "Ayúdanos a Empezar". Esta institución cuenta con 32 años de vida institucional formando y ayudando a niños y jóvenes con discapacidades especiales que asisten a preparase a diario a este centro educativo. Se acudido hasta los predios de esta institución para realizarle una entrevista a los docentes que laboran en esta unidad educativa con el fin de hacerles preguntas específicas relacionadas con la problemática. A continuación se detallan las preguntas realizadas en la entrevista a la docente de la Unidad educativa "Ayúdanos a empezar".
¿Ustedes como docentes reciben capacitaciones pedagógicas por parte del ministerio de educación para impartir clases a los estudiantes con discapacidad que asisten a la unidad educativa?

Los docentes de estas unidades especializadas si reciben capacitaciones por parte del ministerio de educación, pero las nuevas autoridades de no toman en cuenta a la educación especial, estas unidades están siendo evaluadas y sin embargo los docentes tienen queja por parte de las evaluaciones ya que son evaluados como docentes regulares y con como docentes en educación especial. Sin embargo el desarrollo de la prueba para los docentes de educación especial, está siendo realizado por docentes internacionales especializados en educación especial. Esto es algo contradictorio puesto a que si comparamos la realidad de la educación especial en nuestro país con países del exterior.

\section{¿Cree usted que el programa/pensum de estudio para los estudiantes con discapacidades especiales es adecuado?}

El pensum de las unidades educativas especializadas es otro factor que se debate mucho, ya que estas instituciones trabajan con el mismo pensum que las unidades regulares, a diferencia de las unidades especializadas que toman la parte funcional del pensum según lo requiera el estudiante especial por ejemplo: la importancia de conocer su país, las carreteras por que permiten transportar los productos, se toma lo funcional porque son funciones básicas que ellos deben de saber para su subsistencia. Además se les enseña actividades de jardinería, arreglo de casa, para que puedan desempeñarse hacer actividades ya que físicamente pueden hacerlas siempre y cuando tengan una vigilancia.

En el caso de la Unidad de Educación especial "Ayúdanos a empezar" el pensum académico que ahora se lo denomina programa funcional, ha sido desarrollado por los mismo profesores de la unidad educativa, pero el ministerio al momento de realizar los informes ubican a la escuela como una unidad educativa regular, hay que recordar que los alumnos especiales necesitan la atención personalizada para su aprendizaje, pero esto no es tomado en cuenta por parte del ministerio de educación, este es un tema importante que debe ser considerado por el ministerio de educación.

\section{¿Las instituciones de educación especial cuenta con los recursos/equipos necesarios para atender y los estudiantes con discapacidad??}

Aquí no se cuenta con, equipos especiales, material de trabajo adecuado para trabajar con los niños con discapacidad. El ministerio de educación no les proporciona un presupuesto para bastecerse de los materiales para la atención de los estudiantes con discapacidades. Tampoco hay arreglos en la infraestructura por parte del gobierno para mejorar la calidad den la educación especial.

Los estudiantes con discapacidades especiales requieren que atención y aprendizaje personalizado, a ellos no se puede dejar solos a los estudiantes con discapacidad ya que pueden ser utilizados por personas mal intencionadas y guiarlos hacer 
ilícitos ya que ellos no tienen noción de lo que hacen por su discapacidad. También el docente debe de estar pendiente de la disciplina del estudiante especial, puesto a que ellos son más vulnerables en este sentido por su pérdida de la noción del tiempo.

\section{¿Cree usted que las nuevas reformas educativas aplicadas desde el 2010 favorecen al sistema de educación especial?}

Desde el 2010 empezaron cabios muy controversiales dentro del sistema educativo y fue en este mismo periodo donde el vicepresidente el Sr. Lenin moreno se dio un gran auge y apoyo a las personas con discapacidad y se notó un poco de ayuda en la educación especial, pero sin embargo desde su salida nuevamente este tema ha quedado en el olvido. Sin embargo a pesar de él las reformas incluyan los deberes y derechos de las personas con discapacidad y las facilidades que a estos chicos se les debe de ofrecer, si asistimos a la realidad esto no sucede ya que falta inversión en estas instituciones especiales.

\section{ANÁLISIS Y DISCUSIÓN}

Esta investigación tiene como fin puntualizar aspectos importantes sobre la problemática basada en los datos recopilados en la entrevista y dar a conocer al ministerio de educación cuales son las necesidades de los docentes en las unidades de educación especial, cuáles son sus requerimientos para poder brindar un buen aprendizaje a los niños y jóvenes con discapacidades especiales en nuestro país. A continuación se citan puntos muy importantes que deben ser más que tomados en cuenta implementados para mejorar la educación especial:

- Es necesario que se realice cambio dentro del plan de estudios o pensum que maneja el ministerio de educación, con el fin de que se experimenten cambios en las metodologías de enseñanza en los estudiantes con discapacidades especiales.

- El ministerio de educación debe gestionar un presupuesto o financiamiento para las unidades educativas especializadas con el fin de proporcionar el mobiliario adecuado para que los estudiantes especiales puedan recibir sus clases y a los profesores se les facilite poder prepararlos.

- Debe realizarse evaluaciones coherentes por parte del ministerio de educación, tanto a las unidades educativas como a los docentes que se dedican a preparar a estudiantes con discapacidades especiales, y que estos son evaluados como docentes regulares.

- Muchos de los estudiantes vienen del campo y muchas veces no tienen los recursos para asistir a la escuela a preparar sin embargo el ministerio con sus constantes controles no comprenden la situación de las familias que tienen niños con discapacidades especiales.

\section{CONCLUSIONES}

La reforma de la educación ecuatoriana, ha sido creada buscando contribuir con una función social de trascendencia a la actual sociedad denominada del conocimiento. El propósito de la reforma educativa está orientado a la educación de calidad, pertinencia y calidez sustentada en valores, que conlleve a la formación de la persona, no solo para el trabajo y la competencia, sino y sobre todo, para el buen vivir en comunidad. Se ha insertado en la agenda nacional y en las políticas educativas el tema de la inclusión para las personas con discapacidad y para todos los grupos vulnerables. El Modelo Educativo Ecuatoriano debe construirse con un enfoque inclusivo basado en los Derechos Humanos. En las diferentes dependencias del Ministerio de Educación hay una toma de conciencia de que la educación de las personas con discapacidad es responsabilidad de todo el sistema educativo. Sin embargo esto queda solo empapeles al nivel del ministerio de educación, es verdad que los procesos han indo cambiando y se está corrigiendo la situación de a poco, pero se está dejando afuera la educación especial, el cambio se está produciendo el termino globalizado dejando afuera la educación especial, que necesita sus propias reformas, su plan funcional y muchas otros requerimiento que deben ser tomados en cuenta por parte del ministerio de educación. Debemos de concientizar y proporcionar ayuda a la educación especial, puesto que ellos también tienen el derecho a recibir una educación de calidad y con calidez.

\section{BIBLIOGRAFÍA}

Almeida, L. (2012). Proyecto de adaptación curricular del modelo de capacitación del Programa Ramón Ramón Arregui Monreal para incluir a jóvenes desde los 12 años Tesis.

Constitución del Ecuador (2012). Constitución del Ecuador 2012. Registro Oficial, 449(Principios de la participación Art.):67.

Educación Especial e Inclusiva - Ministerio de Educación (2015). Educación Especial e Inclusiva - Ministerio de Educación.

González, M. T. G. (2008). Diversidad e inclusión educativa: algunas reflexiones sobre el liderazgo en el centro escolar. REICE: Revista Electrónica Iberoamericana sobre Calidad, Eficacia y Cambio en Educación, 6(2):82-99.

María, M., Ortega, S., and Zoraida, M. (2015). Educación Inclusiva: Realidad y Desafios.

Ministerio de Educación (2007). Instituciones de Educación Especial - Ministerio de Educación.

Montenegro, R. d. P. and Tigre, M. A. (2013). "Rol de los docentes en la inclusión educativa". Tesis de Pregrado, page 51 .

Pombo, M. F. et al. (2015). Reforma educativa en ecuador y su influencia en el clima organizacional de los centros escolares. Observatorio de la Economía Latinoamericana, (209). 